\title{
Tailored Mechanical Properties and Residual Stresses of a-C:H:W Coatings
}

\author{
C. Schmid ${ }^{1, a^{*}}$, H. Hetzner, ${ }^{2, b}$, S. Tremmel ${ }^{2, ~ c}$, F. Hilpert $^{3}$ and K. Durst ${ }^{1, d}$ \\ ${ }^{1}$ Technische Universität Darmstadt, Physical Metallurgy (PhM), \\ Alarich-Weiss-Straße 2, 64287 Darmstadt, Germany \\ ${ }^{2}$ Friedrich-Alexander-Universität Erlangen-Nürnberg, Chair of Engineering Design (KTmfk), \\ Martensstraße 9, 91058 Erlangen, Germany \\ ${ }^{3}$ Friedrich-Alexander-Universität Erlangen-Nürnberg, General Materials Properties, \\ Martensstraße 5, 91058 Erlangen, Germany \\ ac.schmid@phm.tu-darmstadt.de, ${ }^{b}$ hetzner@mfk.fau.de, ${ }^{c}$ tremmel@mfk.fau.de, \\ dk.durst@phm.tu-darmstadt.de \\ ${ }^{*}$ corresponding author
}

Keywords: a-C:H:W, deposition parameters, tailored properties, residual stress, focused ion beam, digital image correlation

\begin{abstract}
In this study, three different a-C:H:W coatings with predefined hardness values, ranging from 10 up to $16 \mathrm{GPa}$, were deposited by selecting suitable parameter set points and adjusting substrate bias voltage of the reactive unbalanced magnetron sputtering process according to a previously created regression model. To obtain this model, the four main process parameters had been varied according to a central composite design and their influence on the mechanical properties of the a-C:H:W coating had been investigated previously by nanoindentation. The three coating variants of this study were investigated in terms of microstructure, mechanical properties and residual stresses. It turned out, that by the use of the regression model, indeed a-C:H:W coatings of tailored mechanical properties can be deposited. Residual stresses were measured by means of focused ion beam milling of a double-slit geometry, which causes the internal stresses to relax, and mapping of the resultant relief strain by digital image correlation. A nearly linear relation between the applied bias voltage and the hardness, the modulus of elasticity of the coating as well as the determined relief strain was observed. Thus, residual stresses of the coatings increase disproportionately with applied bias voltage. The obtained results can be helpful for tailored coating design and further optimization of a-C:H:W coatings.
\end{abstract}

\section{Introduction}

Amorphous carbon coatings are well known for their beneficial properties such as high hardness combined with low coefficients of friction, chemical inertness and high wear-resistance [1]. However, these coatings frequently suffer from high internal stresses and thus show poor adhesion to many technical relevant substrates like steel, limiting their fields of application. Therefore, different adhesive interlayers were developed for enhancing the adhesion of the coatings [2-4]. Additionally, amorphous carbon coatings, such as hydrogenated amorphous carbon $(\mathrm{a}-\mathrm{C}: \mathrm{H})$, can be doped with transition metals to modify specific properties [5]. In this context, one major advantage of metal modified hydrogenated amorphous carbon (a-C:H:Me) coatings is the reduced internal stresses and the associated enhanced effective adhesion of the coatings to the substrate [6,7]. The probably most common type of a-C:H:Me is tungsten modified hydrogenated amorphous carbon (a-C:H:W).

Typically, a-C:H:W coatings can be regarded as nano-composite materials consisting of a hydrogenated amorphous carbon $(\mathrm{a}-\mathrm{C}: \mathrm{H})$ matrix with embedded nanometer clusters of tungsten carbide [7-9]. These coatings are commonly deposited by reactive magnetron sputtering of a $\mathrm{W}$ or WC target in hydrocarbon atmosphere [6, 8-11]. Therefore, structural as well as mechanical properties of a-C:H:W can be modified within a wide range by variation of process parameters 
during deposition [6, 11-14]. These aspects make a-C:H:Me in general very suitable candidates for tailored coatings with the capability to optimize their properties for specific applications [7].

The present study is based on previous work, in which key process parameters were varied by the use of design of experiments. Moreover, not only the resultant coating properties as a function of applied process parameter were investigated, but also a quadratic regression model was derived by statistical evaluation of the experimental data, which is expected to enable deposition of coatings with predefined i.e. tailored mechanical properties. In the present study, three a-C:H:W coatings of predefined hardness were deposited by adjusting bias voltage according to the regression model. The obtained coatings were investigated in terms of mechanical properties and residual stresses. Here, residual stresses were determined by a combination of focused ion beam (FIB) milling and digital image correlation (DIC) enabling the measurement of residual stresses of coatings regardless of whether they are amorphous or crystalline [15] - on substrates of technical relevance like steel substrates.

\section{Experimental details}

Deposition of coatings Three different a-C:H:W coatings were deposited onto polished (final polishing, diamond slurry $1 \mu \mathrm{m})$ disks $(\varnothing 30 \mathrm{~mm} \times 5 \mathrm{~mm})$ of cold work tool steel 1.2379 hardened and tempered to HRC 62. Prior to deposition of the coatings, the substrates were cleaned (ultrasonic bath, acetone and isopropanol) and plasma etched inside the coating machine. The a-C:H:W coatings were deposited by reactive unbalanced magnetron sputtering of a binder-free WC target in argon-ethine atmosphere using an industrial coating equipment (H-O-T, Germany) with threefold rotating substrate holder. Sufficient adhesion of the functional coating was ensured by deposition of an Cr- and WC-based adhesion layer by arc evaporation and sputtering of a $\mathrm{Cr}$ and binder-free WC target, respectively. For achieving coatings with predefined mechanical properties, specific process parameters obtained with a methodology similar to that presented in [16] were used. The essential approach is given in the following:

First, the influence of the main process parameters, namely cathode power $P_{\text {sputter }}$, argon $\Phi(A r)$ and ethine $\Phi\left(\mathrm{C}_{2} \mathrm{H}_{2}\right)$ flow rate, at variable total system pressure, and negative substrate bias voltage $U_{\text {bias }}$ on the mechanical properties in terms of hardness and modulus of elasticity of the a-C:H:W coating was investigated. For a systematical evaluation of the single effects, the selected parameters were varied according to a central composite design comprising a total of 25 different parameter combinations. The resulting mechanical properties of the coating as hardness and modulus of elasticity were subsequently determined by means of nanoindentation. Further, coating thickness was measured by the calotte grinding method. The obtained results were statistically evaluated using the statistics software Minitab16 (Minitab, UK). Effects caused by variation of one individual parameter as well as two-factor interactions were considered in the analysis. It was found that $\Phi\left(\mathrm{C}_{2} \mathrm{H}_{2}\right)$ and $U_{\text {bias }}$ have the strongest influence on the hardness of the coating. Consequently, a regression model that describes the relation between specific coating properties and the process parameters $\Phi\left(\mathrm{C}_{2} \mathrm{H}_{2}\right)$ and $U_{\text {bias }}$, at a given $P_{\text {sputter }}$ and $\Phi(\mathrm{Ar})$, was derived from the results. Fig. 1 exemplarily shows the contour plot giving the relation between $\Phi\left(\mathrm{C}_{2} \mathrm{H}_{2}\right)$ and $U_{\text {bias }}$ and the hardness of the coating. Additionally, a regression analysis of deposition rate was performed to be able to adapt the resultant a-C:H:W layer thickness.

In a next step, three coating systems CS1-CS3 with desired hardness values were selected (see Fig. 1) and the required process parameters were determined from the regression model. Table 1 shows the parameters used for deposition of the a-C:H:W layer and the corresponding hardness values predicted by the model $H_{\text {model }}$. Furthermore, the deposition time was adjusted in such a way that the expected thickness of the a-C:H:W layer is about $1.5 \mu \mathrm{m}$. 


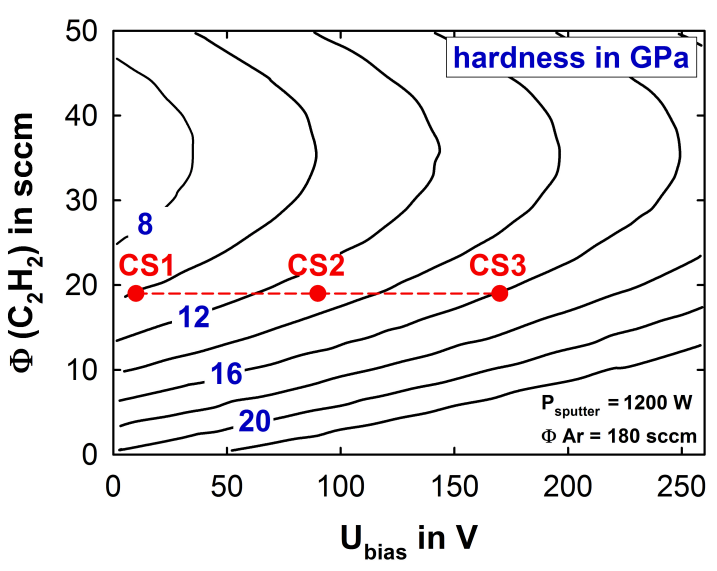

Table 1: Process parameters, determined on basis of the regression model, for the deposition of the coating modifications CS1-CS3 with predefined hardness values.

\begin{tabular}{lccc}
\hline coating & $\begin{array}{l}\Phi\left(\mathrm{C}_{2} \mathrm{H}_{2}\right) \\
\text { in sccm }\end{array}$ & $\begin{array}{c}U_{\text {bias }} \\
\text { in V }\end{array}$ & $\begin{array}{c}H_{\text {model }} \\
\text { in GPa }\end{array}$ \\
\hline CS1 & 19 & 10 & 10 \\
CS2 & 19 & 90 & 13 \\
CS3 & 19 & 170 & 16 \\
\hline
\end{tabular}

Fig. 1: Regression model describing the relation between the hardness of the coating and the process parameters $\Phi\left(\mathrm{C}_{2} \mathrm{H}_{2}\right)$ and $U_{\text {bias }}$ for a given $P_{\text {sputter }}=1200 \mathrm{~W}$ and $\Phi(A r)=180$ standard cubic centimeters per minute $(\mathrm{sccm})$. Additionally, the positions of the selected coating modifications CS1-CS3 are given.

Characterization of the coatings Microstructure and thickness of the a-C:H:W coatings were obtained by focused ion beam (FIB) cross-sections and scanning electron microscopy (SEM) with a Zeiss Crossbeam 1540 (Carl Zeiss, Germany). As a first step a platinum protection bar was deposited on top of the coating to enhance the quality of the final cross-section. Subsequently the cross-section was milled with decreasing ion currents (final milling, $100 \mathrm{pA}$ at $30 \mathrm{kV}$ ) and imaged by SEM.

Hardness $H$ and modulus of elasticity $E$ of the coatings were determined by means of nanoindentation with a nanoindenter XP (MTS Nano Istruments, USA) equipped with a three-sided Berkovich diamond tip. The indentations were performed using continuous stiffness measurement (CSM) technique with standard parameters $(45 \mathrm{~Hz}, 2 \mathrm{~nm})$ and mechanical properties, $H$ and $E$, were evaluated from load-displacement data according to the Oliver-Pharr method [17]. Here, a Poisson's ratio of 0.3 is assumed for the coatings. The mechanical properties were determined in a depth range between $100-150 \mathrm{~nm}$ where a constant and depth independent modulus was observed. A total of 25 indents per coating were performed. The distance between the individual indents was 15 $\mu \mathrm{m}$.

Residual stress levels in the coatings were determined by means of a combination of FIB milling and digital image correlation (DIC) according to the procedure described in [18]. The method is based on the relaxation of internal residual stresses by FIB milling of a double-slit geometry and mapping of the resulting displacements in between the two slits by DIC. Therefore, two high resolution SEM images of the area of interest, one before and one after the FIB milling procedure, are taken. Finally, the residual stresses can be calculated from the determined relaxation strain and the elastic properties of the coating, e.g. obtained from nanoindentations. In the literature different relaxation geometries, like e.g. a single slot [15], annular trenches or pillars $[19,20]$ are proposed. To enable simple calculation of residual stresses by use of Hooke's law, the applied milling geometry, depth $d$ and distance $w$ between the two slits, was previously optimized with regard to the coating thickness $t$. However, distance $w$ is also restricted by the capability of FIB milling and mapping of resulting displacements by DIC. Here, the length of the double-slits was ca. $25 \mu \mathrm{m}$ with a distance between them of $2.5 \mu \mathrm{m}$ and the milling depth was set $d>t$. This approach was exemplarily validated by finite element simulations with the commercial software suite ABAQUS (Dassault Systèmes, France), revealing a maximum mismatch of less than $\approx 5 \%$ between both determined residual stress values. Further information about proper relaxation geometry can be found elsewhere [21]. Moreover, for evaluation of residual stresses of the coatings, an equibiaxial stress state is assumed. FIB milling of the double-slit geometry was conducted by an automated milling procedure, which has been optimized to reduce FIB damage and to attain high milling accuracy. The procedure encloses several milling steps with decreasing ion currents (acceleration voltage $30 \mathrm{kV}$ ) ranging from $500 \mathrm{pA}$, for coarse milling, down to $100 \mathrm{pA}$ for the final milling step. The resulting displacement gradients were mapped with the commercial DIC software Veddac 6.1 (Chemnitzer Werkstoffmechanik, Germany) setting the correlation parameters with regard to the 
image quality and possible offset between both images. Since the coatings inherently showed sufficient topographic features for highly resolved DIC, previous decoration of the coating surface, e.g. with nanoparticles, was not necessary. A total of 5 measurements per coating were conducted for residual stress evaluation.

\section{Results}

Microstructure Fig. 2 shows FIB cross-sections of the coating modifications CS1-CS3 revealing the basic structure consisting of adhesive layer and a-C:H:W functional layer. As can be seen, the architecture and layer thickness of the adhesive layer is the same for all three coatings. The thickness of the Cr layer, the Cr-WC gradient and the WC layer is roughly $30 \mathrm{~nm}, 50 \mathrm{~nm}$ and $100 \mathrm{~nm}$, respectively. This gives evidence for a high reproducibility of the used deposition process, as the adhesive layers were deposited with same process parameters within each batch. With adjusting the deposition time according to the regression model, also the thickness of the different a-C:H:W layers is equal. Here a value of about $1.6 \mu \mathrm{m}$ is measured, indicating a high reliability of the derived regression model.

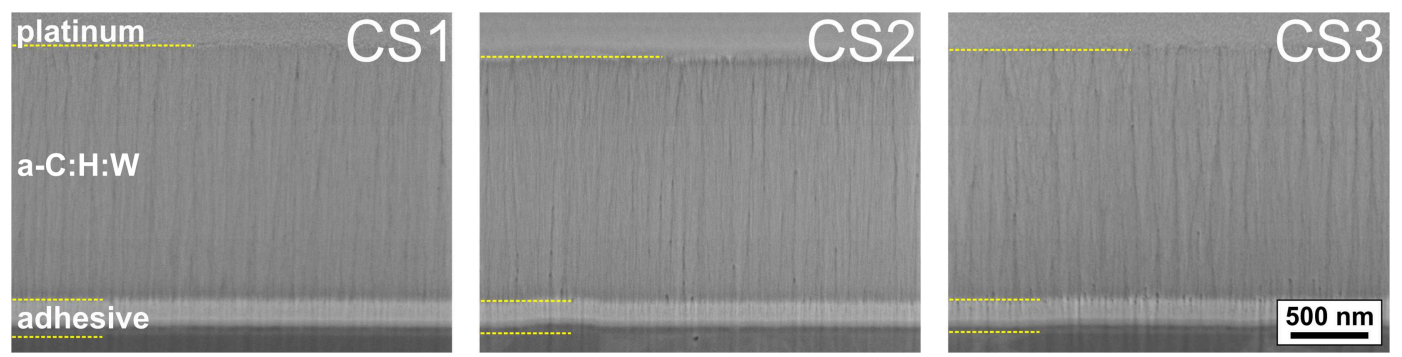

Fig. 2: FIB cross-sections of the coatings CS1-CS3 revealing equal thickness of the a-C:H:W functional layer of about $1.6 \mu \mathrm{m}$. No significant differences are obvious between the microstructure of the coatings.

Further, all three a-C:H:W coatings show a weak columnar microstructure. With increasing $U_{\text {bias }}$ from $10 \mathrm{~V}$ up to $170 \mathrm{~V}$, no significant differences in microstructure of the coatings can be ascertained. The columnar microstructure is typical for this type of coatings and is discussed to be responsible for the reduction of residual stresses [13].

Mechanical properties and validation of regression model The hardness and modulus of elasticity of the coatings were measured in a depth range between $100-150 \mathrm{~nm}$ by nanoindentation using CSM technique. Fig. 3 shows the measured hardness of the three different coatings in comparison with hardness values predicted by the regression model (see Table 1). As can be seen from Fig. 3, the obtained mechanical properties of coating CS1-CS3 correspond well to the predicted values. The generated regression model thus allows deposition of a-C:H:W coatings of defined hardness. The coatings CS1-CS3 show hardness values between $9.4 \mathrm{GPa}$ and $14.8 \mathrm{GPa}$ and related moduli of elasticity between 117 - $172 \mathrm{GPa}$, respectively (see Table 2). So, hardness as well as modulus of elasticity increase considerably with $U_{\text {bias }}$. The relative strong scattering of the measurements derive from the columnar microstructure and surface defects, which occur on top of the coatings and lead to an increased surface roughness. These defects are probably caused by droplets, which arose during cathodic arc deposition of the $\mathrm{Cr}$ adhesion layer. An increase of $H$ and $E$ with bias voltage of a-C:H:W deposited by unbalanced magnetron sputtering in an argon-ethine atmosphere was also reported by Strondl et al. [6] and Pujada and

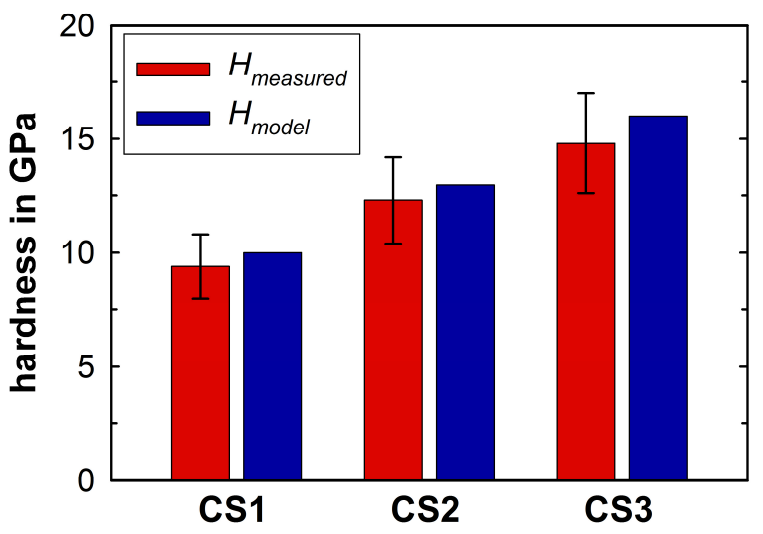

Fig. 3: Hardness of coating systems CS1-CS3 measured by nanoindentation in comparison with predicted hardness values given by the regression model. 
Residual stresses In addition to mechanical properties, especially residual stresses are crucial for a reliable use of coatings and thus are an important aspect for tailored coating design. Fig. 4 shows exemplarily one FIB milled double-slit geometry of each coating superimposed with the resulting displacement gradient obtained by DIC. A linear and symmetric gradient across the remaining bar between the two slits with maximum displacements at the edges is found. Since the bars expand, coatings are subjected to residual compressive stresses, which is typical for a-C:H:W $[11,14]$. The unusual fluctuations in the relaxation displacements indicate an influence of the columnar microstructure on the relaxation gradient. Comparing all three gradients, it becomes evident that the resultant displacements increase from CS1 to CS3, i.e. with increasing $U_{\text {bias }}$. Additional to the gradients, the corresponding displacement vs. position plots of all five measurements per coating system are shown. The slopes of linear regression of the displacement vs. position plots $(d u / d x)$ give the respective relief strain $\varepsilon_{\text {rel. }}$.
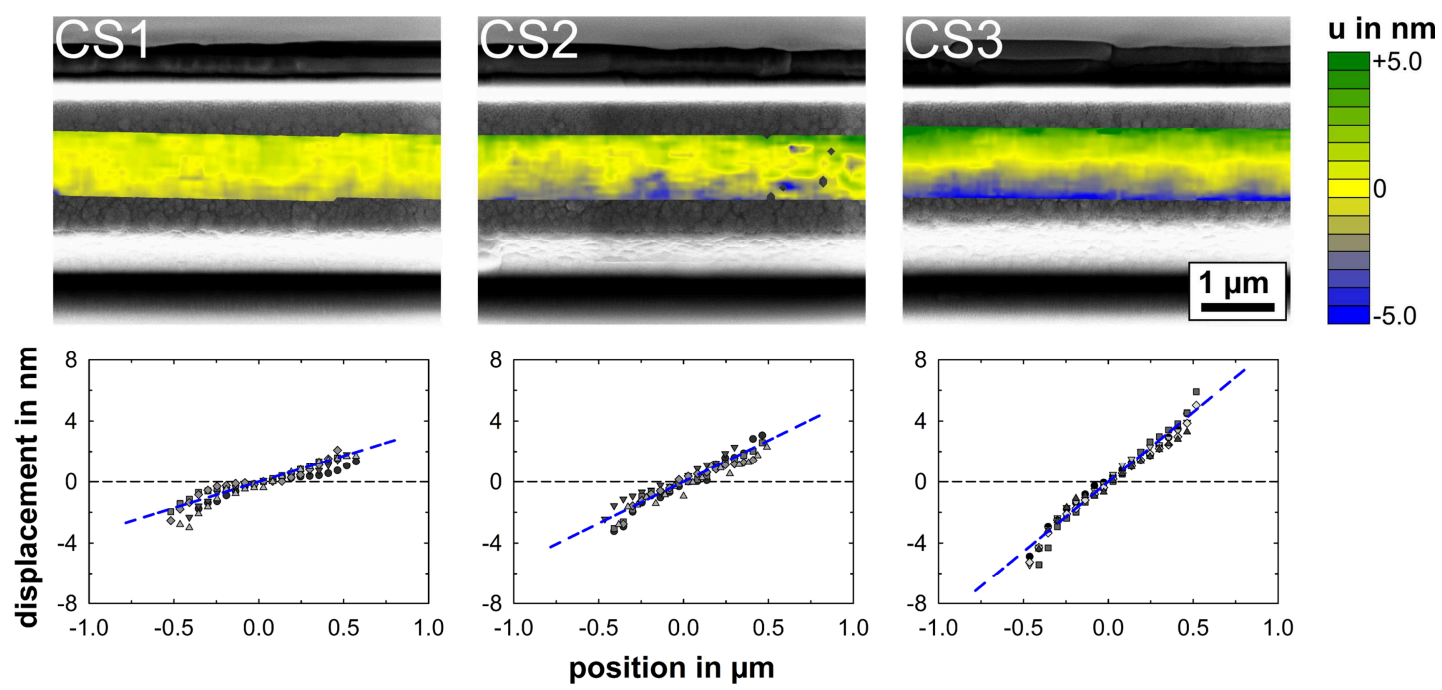

Fig. 4: Representative displacement gradients of coating CS1-CS3 obtained by DIC and corresponding displacement vs. position plots of all five measurements per coating system for the evaluation of relief strain (slope of linear regression).

As already indicated by the displacements, the relief strain also increases considerably with increasing $U_{\text {bias }}$. For the coatings CS1-CS3, relief strain increases from $0.34 \%$ to $0.92 \%$. From the relief strain the residual stresses of the coatings were calculated using the modulus of elasticity determined by nanoindentations. This simple approach is valid for the given geometry within an error of less than the overall measurement uncertainty as verified by finite element analysis. For the coatings CS1-CS3, residual stress values in the range between $-0.40 \mathrm{GPa}$ and $-1.57 \mathrm{GPa}$ are obtained. Increasing residual compressive stresses of a$\mathrm{C}: \mathrm{H}: \mathrm{W}$ with increasing bias voltage were also reported elsewhere [6, 11]. Table 2 summarizes the determined properties of the coatings.

Table 2: Comparison of mechanical properties, relief strain and residual stresses of the coatings.

\begin{tabular}{lcccc}
\hline coating & $H$ in $\mathrm{GPa}$ & $E$ in $\mathrm{GPa}$ & $\varepsilon_{\text {rel. }}$ in $\%$ & $\sigma_{\text {res. }}$ in $\mathrm{GPa}$ \\
\hline $\mathrm{CS} 1$ & $9.4 \pm 1.4$ & $117 \pm 12$ & $0.34 \pm 0.06$ & $0.40 \pm 0.07$ \\
$\mathrm{CS} 2$ & $12.2 \pm 1.0$ & $145 \pm 13$ & $0.54 \pm 0.07$ & $0.79 \pm 0.11$ \\
$\mathrm{CS} 3$ & $14.8 \pm 2.2$ & $172 \pm 20$ & $0.92 \pm 0.10$ & $1.57 \pm 0.18$ \\
\hline
\end{tabular}

\section{Discussion}

The obtained dependencies of $H, E, \varepsilon_{\text {res. }}$ and $\sigma_{\text {res. }}$ on the applied bias voltage are shown in Fig. 5. As can be seen, $H$ and $E$ of the three coatings increase directly proportional to applied bias voltage. As $H$ and $E$ increase to the same extent, a nearly constant $H / E$ ratio with values between $0.080-0.086$ for all three coatings is obtained. The $H / E$ ratio is also an important design parameter for coatings especially under tribological load and is regarded as a measure for the elastic strain to failure [22]. As a consequence, independent adjustment of hardness and modulus of elasticity of the 
coatings by adaption of $U_{\text {bias }}$ according to the regression model seems to be possible only to a very limited extent.

The relief strain obtained by DIC increases nearly linear from $0.39 \%$ at $10 \mathrm{~V}$ up to $0.90 \%$ at $170 \mathrm{~V}$ bias voltage. With milling depth $d$ of the double-slits exceeds the coating thickness, the observed relief strain on top of the coatings reflects the average residual stress state of the entire coating system, neglecting any possible stress gradients. Moreover, owing to high defect density on the coating's surface, it was hard to avoid having any defects in the vicinity of the milled doubleslits. However, Sebastiani et al. [23] showed by incremental FIB milling on chromium nitride coatings

produced by cathodic arc evaporation, that such defects do in fact influence the stress gradient but not the average stress present within the entire coating. Since the used relaxation geometry is only sensitive for residual stresses acting in one direction, i.e. perpendicular to the slits, an equibiaxial stress state of the coatings is assumed.

As can be seen from Fig. 5, residual stresses of the coating considerably increase from $-0.40 \mathrm{GPa}$ at a bias voltage of $10 \mathrm{~V}$ up to $-1.57 \mathrm{GPa}$ at $U_{\text {bias }}$ of $170 \mathrm{~V}$. Since the magnitude of residual stress is given by the magnitude of modulus of elasticity as well as the observed relief strain, both increase nearly linear with $U_{\text {bias }}$, the residual stress level of the coatings actually increase disproportionately with bias voltage $\left(\sigma_{\text {res. }} \sim\left(U_{\text {bias }}\right)^{2}\right)$. Beside the undesirable aspects of residual stresses in coating systems, they also can be advantageous to a certain extent under specific loading conditions. Schaufler et al. observed for well adhering coatings [24] a strongly enhanced fatigue life time of coated steel substrates, which they attributed to the high residual compressive stress level of the coatings [25]. Moreover, the degradation rate of a-C:H coatings under erosive load could be reduced by increasing the residual compressive stresses of the
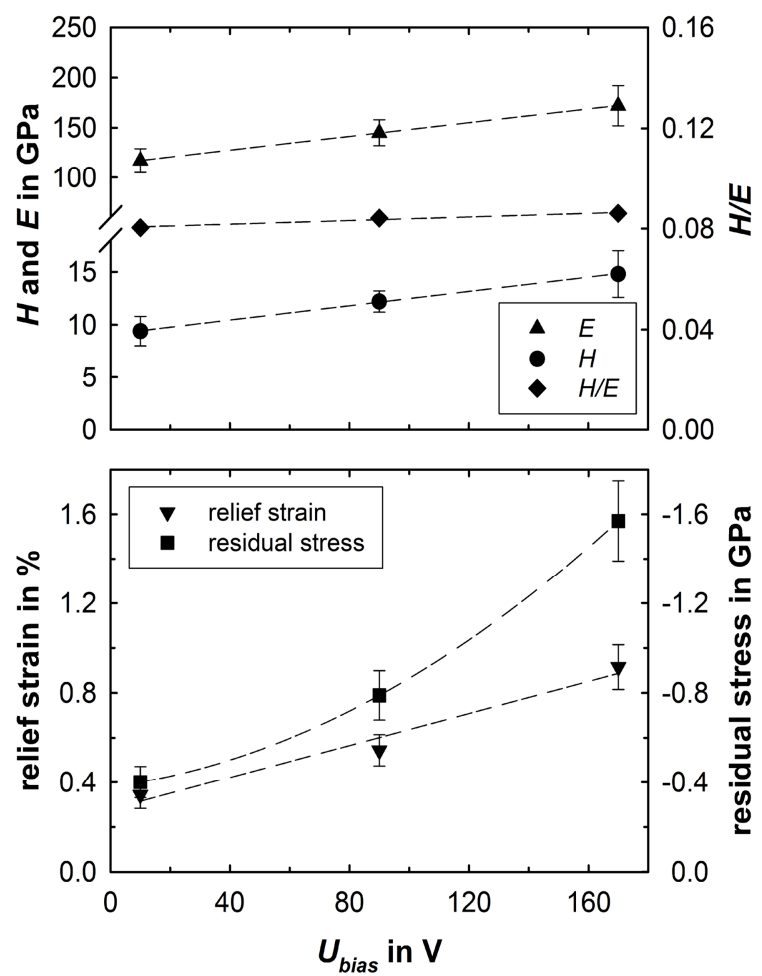

Fig. 5: Correlation between mechanical properties (hardness and modulus of elasticity), observed relief strain and residual stresses of the coatings CS1-CS3 with the applied negative substrate bias voltage. coating [26].

\section{Conclusion}

Three different tungsten modified hydrogenated carbon coatings (a-C:H:W) with hardness values ranging from 9.4 to $14.8 \mathrm{GPa}$ were deposited by reactive unbalanced magnetron sputtering by a variation of negative substrate bias voltage according to a previously derived regression model. By the proposed experimental approach, a-C:H:W coatings of tailored hardness can be deposited. These coatings were further investigated in terms of mechanical properties and residual stresses. Here, a linear relation between hardness as well as modulus of elasticity and applied bias voltage was found. No significant variation of $H / E$ ratio was observed, limiting independent adjustment of hardness and modulus of elasticity. However, it was observed that relief strain due to relaxation of internal stresses by FIB milling also increases nearly linear with bias voltage. Since the magnitude of residual stresses of the coatings depends on both the relief strain and the modulus of elasticity, it increases disproportionately $\left(\sigma_{\text {res. }} \sim\left(U_{\text {bias }}\right)^{2}\right)$ with applied bias voltage.

In future work, chemical composition of the coatings will be analyzed by Auger electron spectroscopy and Raman spectroscopy will be performed to gain structural information. Additionally, two more coating systems CS4 and CS5 with same expected hardness values as of 
CS1 and CS3 will be deposited by varying only $\Phi\left(\mathrm{C}_{2} \mathrm{H}_{2}\right)$ according to the presented regression model. This will be done in such a way that both connecting lines CS1/CS3 and CS4/CS5 intersect in CS3 by setting $U_{\text {bias }}$ fix to $90 \mathrm{~V}$. Here, a higher ethine flow rate should lead to a higher portion of a-C:H matrix $[10,14]$ and thus probably will influence the $H / E$ ratio. Furthermore, it is of interest whether and to what extent the residual stresses can be adjusted independently of $H$ and $E$.

\section{Acknowledgements}

The authors gratefully acknowledge the funding of the German Research Council (DFG) within the DFG project Du-424/7-1 and the scope of the Transregional Collaborative Research Centre on sheet bulk metal forming (SFB/TR 73) in the subproject B4. Furthermore, the authors acknowledge the fruitful discussions within the iSTRESS project. Most of the experimental work was performed at the Institute of General Materials Properties at FAU Erlangen-Nürnberg.

\section{References}

[1] J. Robertson, Diamond-like amorphous carbon, Mater. Sci. Eng. R 37 (2002) 129-281.

[2] K.-R. Lee, K.Y. Eun, I. Kim, J. Kim, Design of W buffer layer for adhesion improvement of DLC films on tool steels, Thin Solid Films 377-378 (2000) 261-268.

[3] M. D. Bentzon, K. Mogensen, J. Bindslev Hansen, C. Barholm-Hansen, C. Træholt, P. Holiday, S. S. Eskildsen, Metallic interlayers between steel and diamond-like carbon, Surf. Coat. Technol. 68/69 (1994) 651-655.

[4] C.-C. Chen, F.C.-N. Hong, Interfacial studies for improving the adhesion of diamond-like carbon films on steel Appl. Surf. Sci. 243 (2005) 296-303.

[5] Association of German Engineers (VDI), VDI guideline 2840, VDI, Düsseldorf, 2005.

[6] C. Strondl, N.M. Carvalho, J.Th.M. De Hosson, T.G. Krug, Influence of energetic ion bombardment on W-C:H coatings deposited with W and WC targets, Surf. Coat. Technol. 200 (2005) 1142-1146.

[7] J.C. Sánchez-López, A. Fernández, Doping and alloying effects on DLC coatings, in: C. Donnet, A. Erdemir (Eds.), Tribology of Diamond-Like Carbon Films, Springer, New York, 2008, 311-338.

[8] K.I. Schiffmann, M. Fryda, G. Goerigk, R. Lauer, P. Hinze, A. Bulack, Sizes and distances of metal clusters in Au-, Pt-, W- and Fe-containing diamond-like carbon hard coatings: a comparative study by small angle X-ray scattering, wide angle X-ray diffraction, transmission electron microscopy and scanning tunnelling microscopy, Thin Solid Films 347 (1999) 60-71.

[9] C. Strondl G.J. van der Kolk, T. Hurkmans, W. Fleischer, T. Trinh, N.M. Carvalho, J.Th.M. de Hosson, Properties and characterization of multilayers of carbides and diamond-like carbon, Surf. Coat. Technol. 142-144 (2001) 707-713.

[10] Bewilogua, C.V. Cooper, C. Specht, J. Schröder, R. Wittorf ,M. Grischke, Effect of target material on deposition and properties of metal-containing DLC (Me-DLC) coatings, Surf. Coat. Technol. 132 (2000) 275-283.

[11] B.R. Pujada, G.C.A.M. Janssen, Density, stress, hardness and Young's modulus of W-C:H coatings, Surf. Coat. Technol. 201 (2006) 4284-4288.

[12] C. Corbella E. Bertran, M.C. Polo, E. Pascual, J.L. Andújar, Structural effects of nanocomposite films of amorphous carbon and metal deposited by pulsed-DC reactive magnetron sputtering, Diam. Relat. Mater. 16 (2007) 1828-1834.

[13] C. Corbella, G. Oncins, M.A. Gómez, M.C. Polo, E. Pascual, J. García-Céspedes, J.L. Andújar, E. Bertran, Structure of diamond-like carbon films containing transition metals deposited by reactive magnetron sputtering, Diam. Relat. Mater. 14 (2005) 1103-1107.

[14] C. Strondl, N.M. Carvalho, J.Th.M. De Hosson, G.J. van der Kolk, Investigation on the formation of tungsten carbide in tungsten-containing diamond like carbon coatings, Surf. Coat. Technol 162 (2003) 288-293.

[15] K.J. Kang, N. Yao, M.Y. He, A.G. Evans, A method for in situ measurement of the residual stress in thin films by using the focused ion beam, Thin Solid Films 443 (2003) 71-77. 
[16] H. Hetzner, R. Zhao, S. Tremmel, S. Wartzack, Tribological adjustment of tungsten-modified hydrogenated amorphous carbon coatings by adaption of the deposition parameters, in: K.-D. Bouzakis, K. Bobzin, B. Denkena, M. Merklein (Eds.), Proceedings of the 10th International Conference THE "A" Coatings 2013, Shaker, Aachen, 2013, 39-49.

[17] W.C. Oliver, G.M. Pharr, An improved technique for determining hardness and elastic modulus using load and displacement sensing indentation experiments, J. Mater. Res., 7 (1992) 1564-1583.

[18] M. Krottenthaler, C. Schmid, J. Schaufler, K. Durst, M. Göke, A simple method for residual stress measurements in thin films by means of focused ion beam milling and digital image correlation, Surf. Coat. Technol., 215 (2013) 247-252.

[19] A.M. Korsunsky, M. Sebastiani, E. Bemporad, Residual stress evaluation at the micrometer scale: Analysis of thin coatings by FIB milling and digital image correlation, Surf. Coat. Technol. 205 (2010) 2393-2403.

[20] M. Sebastiani C. Eberl, E. Bemporad, G.M. Pharr, Depth-resolved residual stress analysis of thin coatings by a new FIB-DIC method, Mat. Sci. Eng. A 528 (2011) 7901-7908.

[21] F. Ahmed M. Krottenthaler, C. Schmid, K. Durst, Assessment of stress relaxation experiments on diamond coatings analyzed by digital image correlation and micro-Raman spectroscopy, Surf. Coat. Technol. 237 (2013) 255-260.

[22] A. Leyland, A. Matthews, On the significance of the $H / E$ ratio in wear control: a nanocomposite coating approach to optimised tribological behavior, Wear 246 (2000) 1-11.

[23] M. Sebastiani, M. Piccoli, E. Bemporad, Effect of micro-droplets on the local residual stress field in CAE-PVD thin coatings, Surf. Coat. Technol. 215 (2013) 407-412.

[24] J. Schaufler, C. Schmid, K. Durst, M. Göken, Determination of the interfacial strength and fracture toughness of a-C:H coatings by in-situ microcantilever bending, Thin Solid Films 522 (2012) 480-484.

[25] J. Schaufler, K. Durst, T. Haas, R. Nolte, H.W. Höppel, M. Göken, The influence of hydrogenated amorphous carbon coatings $(\mathrm{a}-\mathrm{C}: \mathrm{H})$ on the fatigue life of coated steel specimens, Int. J. Fatigue 37 (2012) 1-7.

[26] U. Depner-Miller, J. Ellermeier , H. Scheerer, M. Oechsner, K. Bobzin, N. Bagcivan, T. Brögelmann, R.Weiss, K. Durst, C. Schmid, Influence of application technology on the erosion resistance of DLC coatings, Surf. Coat. Technol. 237 (2013) 284-291. 